\title{
Algorithm Selection for Edge Detection in Satellite Images by Neutrosophic WASPAS Method
}

\author{
Romualdas Bausys ${ }^{1}\left[\right.$, Giruta Kazakeviciute-Januskeviciene ${ }^{1}$, Fausto Cavallaro ${ }^{2, *}(\mathbb{D}$ and \\ Ana Usovaite 1 \\ 1 Department of Graphical Systems, Vilnius Gediminas Technical University, Sauletekio al. 11, LT-10223 \\ Vilnius, Lithuania; romualdas.bausys@vgtu.lt (R.B.); giruta.kazakeviciute-januskeviciene@vgtu.lt (G.K.-J.); \\ ana.usovaite@vgtu.lt (A.U.) \\ 2 Department of Economics, University of Molise, Via De Sanctis, 86100 Campobasso, Italy \\ * Correspondence: cavallaro@unimol.it
}

Received: 29 November 2019; Accepted: 6 January 2020; Published: 10 January 2020

\begin{abstract}
Nowadays, integrated land management is generally governed by the principles of sustainability. Land use management usually is grounded in satellite image information. The detection and monitoring of areas of interest in satellite images is a difficult task. We propose a new methodology for the adaptive selection of edge detection algorithms using visual features of satellite images and the multi-criteria decision-making (MCDM) method. It is not trivial to select the most appropriate method for the chosen satellite images as there is no proper algorithm for all cases as it depends on many factors, like acquisition and content of the raster images, visual features of real-world images, and humans' visual perception. The edge detection algorithms were ranked according to their suitability for the appropriate satellite images using the neutrosophic weighted aggregated sum product assessment (WASPAS) method. The results obtained using the created methodology were verified with results acquired in an alternative way-using the edge detection algorithms for specific images. This methodology facilitates the selection of a proper edge detector for the chosen image content.
\end{abstract}

Keywords: edge detection; orthophoto imagery; MCDM; WASPAS; neutrosophic set

\section{Introduction}

Sustainability is the key aspect in managing natural resources, such as forests, water bodies, and agricultural land. Usually, the aerial data for land observation is acquired as aerial images, either from the satellite images or aerial photography. During the last decade, satellite imagery data have been actively used to keep development sustainable.

Acquired aerial images include information about the various objects of interest. The information of interest can be filtered out using edge detection methods, reducing unnecessary information. The environmental projects are usually constructed applying a vector form of land data. Therefore, all raster objects in the satellite images must be converted into the vector ones in the land management projects. Edge discrimination of a particular object for the creation of an edge map is based on the satellite image content features of the textured and non-textured areas, and these features are associated with the visual perception of the image. The texture is represented by the spatial interrelationships of pixels and can be estimated as local changes of luminosity or intensity pattern [1]. Satellite imagery has a wide variety of texture types that represent different target areas: roads, rivers, lakes, land, vegetation, buildings, and more [2]. Many applications for sustainable project management aim to distinguish these areas in grayscale and/or color images, and edge detection has been used as the pre-processing step before the other subsequent stages of data processing [2-5]. 
Vectorization of the objects usually starts by constructing the edge map. Edges identify and localize significant changes in the digital image. These changes may be caused by object boundaries, textural properties, illumination, orientation, depth, or other reasons [6]. The typical edge detection method aims to highlight the important features in the satellite image. The main edge detection methods, for now, are differential, statistical, multiresolution, morphological based approaches, methods of computational intelligence, and other created edge detection methods [6-9]. A particular algorithm responds differently to the given image content, and results are different for the chosen image. Edge detection algorithms have been used in boundary detection, in image segmentation as a pre-processing step, for example, to extract coastlines between regions of land and sea in aerial photos [2], in content-based image retrieval (CBIR) [10], building detection in low contrast satellite images [6] as well as others computer vision-based applications $[4,5]$. Edge detectors significantly reduce the amount of image data and provide the necessary structural information about objects and regions in the satellite image.

One of the crucial tasks is to discriminate features of interest, related to the appropriate image content and represented by various textures, in satellite images using appropriate methods and algorithms. Many authors have analyzed the textural features that humans can discriminate in the images. In [1], textural characteristics such as roughness, regularity, density, directionality, contrast, and coarseness are described. The researchers in [11] mention contrast, coarseness, busyness, complexity, and texture strength. Other authors reused some previous textural features for the analysis of the visual complexity of the textures [12,13]. In [14], images of real-word scenes were used for their complexity evaluation using the number of objects, a variety of colors, clutter, etc. Most of the authors [1,11-13] have studied the visual characteristics of the individual types of textures in the isolated areas of an image. Our selection of subjective visual features of the satellite images is based on the scientists' works [1,11-16], and validated with the set of experiments. This set of visual characteristics has been applied for the edge detection algorithms.

The edge detection process has been evaluated using a number of different criteria with different optimization directions. Therefore, the selection of the edge detection algorithm for the particular image can be accomplished using multi-criteria decision-making (MCDM) methodology. MCDM is an important technique that is used in sustainability engineering [17], as it is applied in a variety of areas: an improvement of image segmentation [18] and edge detection algorithms [7], sustainable urban development [19], ranking of the urban parks according to their safety [20], environment exploration by autonomous mobile robots [21], concentrated solar power (CSP) [22,23], combine heat and power (CHP) [24]. However, the multi-criteria analysis is not widely applied for the selection of the edge detection methods for application-specific images based on their features. The authors of [5] have provided an idea and a framework for the realization of such an opportunity, but neither the selection of criteria nor practical decision-making has been justified and verified. The research dedicated to the application of MCDM methods in image processing is usually concerned with improving edge detection algorithm characteristics [7].

As an alternative to edge detection, deep learning can be used [25]. It is usually applied as a part of image processing algorithms and the improvement of their characteristics [26-28]. The drawback of this technique is the requirement of a huge amount of the initial data [29]. While deep learning is used for edge detection, this paper covers another topic-the selection of optimal methods for edge detection.

We propose and verified a novel set of visual image features to be used in the MCDM framework. We formulate a new MCDM problem dedicated to the adaptive selection of edge detection algorithms governed by visual features of satellite images. Herewith, we performed ranking of edge detection algorithms according to their suitability for the appropriate satellite image type using the neutrosophic WASPAS method.

The guidelines for appropriate MCDM algorithm choice have been well-documented by Guitouni et al. [30]. The WASPAS method is a unique combination of the weighted sum model 
(WSM) and the weighted product model (WPM). Because of its mathematical simplicity and capability to provide more accurate results as compared to WSM and WPM methods, the WASPAS method is an efficient decision-making tool for solving the problem of algorithm selection. The uncertainty of the initial information expressed by linguistic terms is solved using the neutrosophic set environment [31].

The structure of the work is as follows. Section 2 presents a set of criteria and alternatives for a multi-criteria task for selecting edge detection algorithms based on image content, as well as a framework for implementing the task itself. Section 3 describes the MCDM neutrosophic WASPAS method. Section 4 presents the implementation and results of the experiments, the ranking of the edge detection algorithms based on the neutrosophic WASPAS method, and the verification of the ranking. The conclusions and future works are presented in Section 5.

\section{Selection of Edge Detection Algorithms for Satellite Images}

In the proposed methodology, the selection of edge detection algorithms is governed by the visual features of satellite images. The satellite image features like contrast, smoothness, edge density, and others depend on the spatial arrangement and interrelationship of pixel values as it is perceived as changes of gray tones in the intensity pattern. The perceivable local patterns of pixel values can be treated as textures of the image regions. Places of the image, exposing significant changes of the intensity in the neighboring pixels are perceived as edges. Edges can separate regions of the objects in the image, as well as discriminate the elements of textured regions, depending on the features of the image regions and the edge detection methods [9]. We have identified features of the image regions responsible for the perceived edges and the influence of these features on the edge detectors.

\subsection{Subjective Visual Features of the Satellite Image Content}

Orthographic images are of the most importance to the satellite image content analysis since those images form the basis for the environmental projects. Orthographic image or orthophoto is a geometrically corrected satellite image. The orthographic image content features are based on the intensities of pixels. Lightness information influences the perception of real-world images with a higher degree than color [15,32]. In the simplest case, a group of pixels with similar intensity values being close to each other constitute a region. When the region is neighboring the other region with homogeneous intensity values within it, these two regions of different intensity values are separated by the edge. The boundary delineates the area of the region. When the region consists of similar pixel values, it is considered a smooth region. The region consisting of neighboring contrasting pixel values that are close to each other presents a rough intensity pattern and is considered a textured (rough) region. The basic metrics of these regions are energy, homogeneity, entropy, and others [33]. When in the textured region is represented repeating local variations of image intensities, a lot of short edges are distinctive characters of these regions because of high spatial frequencies of intensities, like grass, water waves, foliage, and similar objects.

The textures of the high and low-frequency range are presented in an image simultaneously. We take into account all types of textures because we analyze visual features throughout the image perceivable by humans. We have selected the most characteristic visual features of orthographic images, such as the size of the individual regions, contrast, roughness, density, and the number of different regions [1,11-16].

For the evaluation of the image content features, the digital orthographic images with a pixel resolution of 0.5 feet from across the United States were used from Earth Explorer [34] (see Figure 1).

The objects of our interest are water bodies: rivers, lakes, ponds, and sea. By selecting the appropriate resolution of digital images, we can distinguish separate objects and/or the appropriate areas and their features. More details are available in the higher resolution images as more edges too [35]. With smaller resolution images, larger objects or areas can be distinguished, and small objects make their detection more difficult. If similar small objects are adjacent in orthoimages, they may be perceived as areas of the respective texture (for example, foliage-rough texture). 


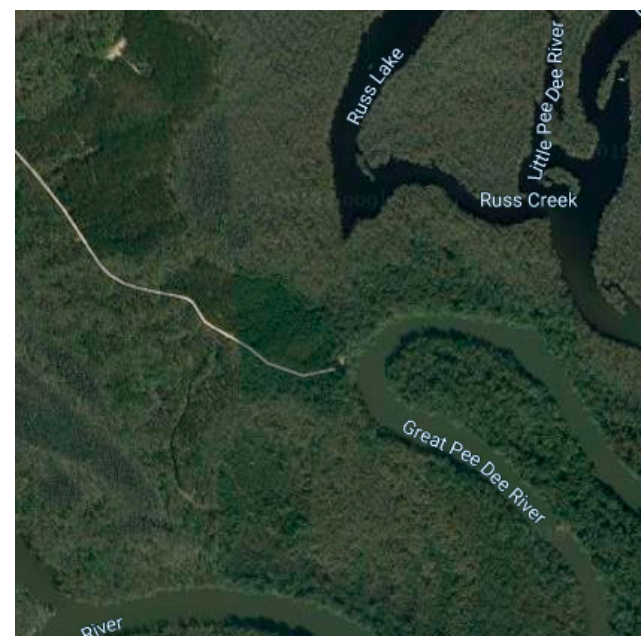

Figure 1. The location view was taken from the satellite map using Earth Explorer (Landsat-7 image courtesy of the U.S. Geological Survey).

The size of the individual regions in the image is closely related to the coarseness of the textured regions. Coarseness [11] is the image region property related to its structure. The textured region is coarser when its elements are larger, and they are less repeated. These regions share the intensity values of the neighborhood pixels and can be characterized by the high local uniformity. By increasing the size of the texture, we can treat its elements as separate objects. With a significant reduction, we have a less coarse texture, and the intensity values of pixels in the neighborhood have low local uniformity.

We have reused this feature to map the overall image structure based on the size of individual regions. Image structure-objects, regions, and edges_-is presented from a bottom-up perspective using pixel intensities, textures, and colors. The larger the regions, the more they are distinguishable from each other.

The "size" criterion of image regions and objects reflects the structural precision of the image. The larger the objects, their elements, and the textural regions, the more precisely boundaries can be defined between them, the easier to distinguish them by the appropriate edge detectors. At the small size, boundaries of the regions tend to merge, and it is problematic to separate them.

Figure 2 illustrates the cropped parts of the analyzed orthographic image with a different size of individual regions. The size of the water area differs significantly from the size of the many surrounding objects (Figure 2a), and the size of the water regions is small (Figure 2b).

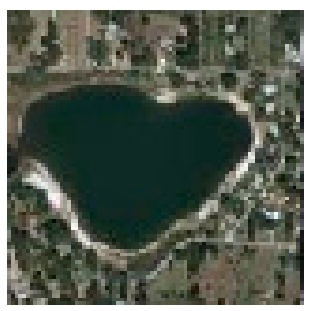

(a)

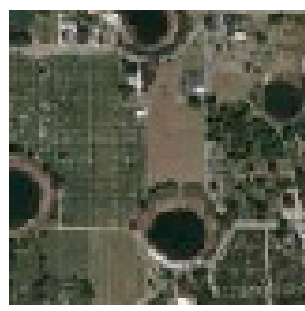

(b)

Figure 2. Water regions and their surrounding (Landsat-7 image courtesy of the U.S. Geological Survey): (a) large water regions with small surrounding elements; (b) small water regions that are surrounded by a high number of different small elements.

Contrast [11] is related more to image quality but not to image structure. Sharp edges can be obtained when the intensity between adjacent regions varies considerably. For textured images with high contrast, the differential edge detectors are not the best choice as no meaningful region will be delineated. In the case of textured regions with high contrast small elements in them, all fluctuations 
of intensity are treated as false edges within the region. Edge detectors, not sensitive to textured areas, can be used $[9,36]$.

Edges will separate the texture's elements if the size of the elements is large (coarse texture), and the intensity of the elements is contrasting. Differential edge operators prefer the values of pixels within the region are as similar as possible. The regions themselves should be smooth with minimal texture. Sharp edges are perceivable in the images with high contrast between regions (see Figure 3a).

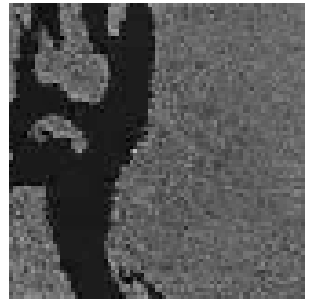

(a)

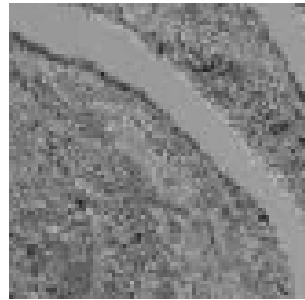

(b)

Figure 3. Contrast between the grayscale image regions (Landsat-7 image courtesy of the U.S. Geological Survey): (a) two regions-river and forest-with high contrast; (b) low contrast between river and forest.

Contrast may be low for a variety of reasons: the artifacts as "motion blur" in the image, adjacent regions have similar pixel values (see Figure 3b).

Roughness is closely related to the busyness feature of the image region [12,13]. The busyness of texture presents the rapid changes of pixel intensity values in neighboring [11]. Roughness represents our feeling of touch. Due to the high spatial frequency of intensity changes, we assume the appropriate visual texture tends to be rough. The low spatial frequency range of intensity changes within the image region makes the region feels smooth. The smooth contrasting regions of satellite images give good results after edge detection using classical edge detection algorithms. In high contrast images, because of natural fluctuations of pixel values, a higher roughness of textured regions is perceived. The part of the smooth water region and the rough forest region (the foliage is visible from above) are presented in Figure 4a,b.

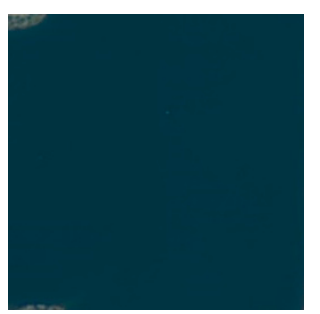

(a)

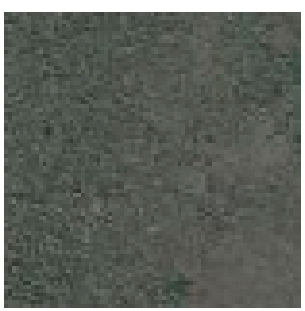

(b)

Figure 4. Roughness feature of different regions (Landsat-7 images courtesy of the U.S. Geological Survey): (a) smooth water region; (b) rough forest region.

Density describes how the fluctuations of intensity are perceived throughout the image between the regions but not only within the regions. Density $[12,13]$ is used to evaluate the sparsity of edges in the image. The density is influenced by the number of elements in the region and the number of regions themselves. The less regions and elements there are in the region, the better the results of the edge detectors. Figure 5a presents the image with low edge density, while Figure $5 b$ presents the image with high edge density. 


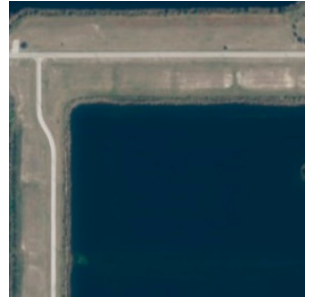

(a)

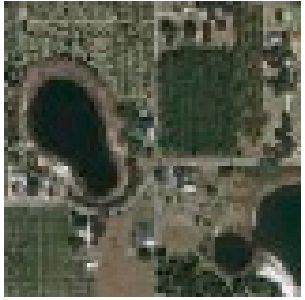

(b)

Figure 5. Density feature of image (Landsat-7 images courtesy of the U.S. Geological Survey): (a) low density; (b) high density.

The number of different regions is based on the intensity of textured and smooth areas [14]. This feature of the satellite image is related to the number of objects in the image and their details. The color feature can be included for respondents besides the intensity to separate regions. There is a correlation between color and intensity, evaluating the complexity of the image, but the color information does not influence the perception significantly [15].

The edge detection algorithms that are sensitive to the textured areas do not produce the precise result for the images which have objects containing many details and complex textures. These algorithms will detect edges of the separated regions if the image has a small number of objects with clearly distinguished details and smooth textures. The edge detection result is more cluttered for the textured surfaces, as edges are derived from the imaginary edges between elements of the texture. For the image containing some regions with similar or identical textures, they will be treated as a single region (Figure 6).

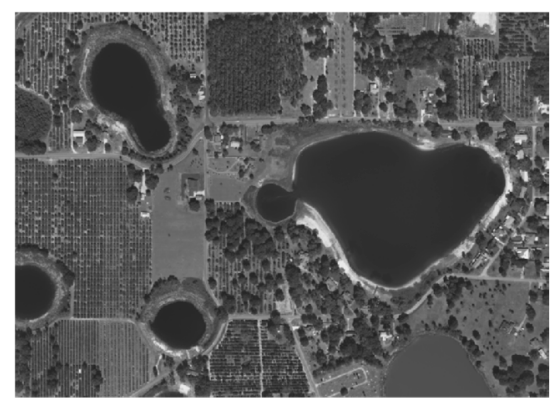

Figure 6. Image contains the number of small and large regions, but different regions with different texture features are only six (Landsat-7 image courtesy of the U.S. Geological Survey).

All five of the above-described image features were selected as our strategy criteria. As the sixth criterion, we have included image processing time. All criteria have a more or less different effect on the result of edge detectors, and time criterion directly influences the duration of edge detection and depends on the algorithm and image size. Therefore, when selecting edge detection algorithms for satellites according to their suitability, it is important to distinguish what visual features of image content have the most significant influence on their suitability, and how important is time.

\subsection{Edge Detection Algorithms and Their Properties Based on the Image Content}

Edge detection is the process which captures the significant changes in the image intensity function. This process should be reliable because the other subsequent stages of satellite data processing depend on it. The input for the process is a discrete image, and the edge map is the output.

There are defined these requirements for proper detection of edges [6]: the optimal edge detector has to find the real edges without noise and other artifacts; the detected edges have to be close to the real (true) edges as much as possible; for the true edge one point for each edge point must be returned. 
We selected the classical edge detection algorithms-Prewitt [37], Kirsch [38], Canny [39], Laplacian of Gaussian (LoG) [40] alongside with Canny-mathematical morphology-based [36] and SubPixel methods [41]—for the implementation of our strategy. The differential edge detectors (Prewitt, Kirsch, Canny, Laplacian of Gaussian (LoG)) belong to first or second-order derivatives operators. The morphological method uses the Canny algorithm and mathematical morphology. SubPixel algorithm for edge detection uses the hypothesis of the partial area effect. These selected edge detection algorithms form the set of alternatives for MCDM problem formulation.

Changes in brightness over a set of pixels refer to the image gradient. The approximation of image gradients can be calculated by convolution using different kernels. The gradient kernel influences the result of edge detection.

The first-order derivative operators-Prewitt, Kirsch, Canny-compute edge gradients of a discrete image in the appropriate directions. The strength and direction of the edge can be evaluated from gradient information by the gradient magnitude and angle of orientation. The edge direction is perpendicular to the direction of the gradient. After thresholding operation, all pixels with gradient values large the defined threshold become possible edge pixels. The morphological operation thins the possible edges to the one point for each edge point. The Canny edge detector is a multistage algorithm and evaluates more nuances in the edge detection process. Morphological edge detection is related to the first-order derivative edge detectors as in first stage, it uses the base of the Canny algorithm. The SubPixel algorithm differs from the differential edge detection algorithms.

Prewitt and Kirsch operators have $3 \times 3$ convolution kernels and average out the small intensity fluctuations of image. The convolution kernels of the Prewitt operator are directed towards the filtering of horizontal and vertical edges. Prewitt's algorithm uses the integer-valued filters and is computationally inexpensive.

Kirsch edge detector uses eight $3 \times 3$ directional convolution kernels. The single kernel is rotated in 45 degrees to get $\mathrm{N}, \mathrm{NW}, \mathrm{W}, \mathrm{SW}, \mathrm{S}, \mathrm{SE}, \mathrm{E}$, and NE compass orientations. Kirsch algorithm, like the other algorithms based on compass kernels, finds the maximum absolute gradient magnitude across all directions and uses it as the edge gradient. Kirsch algorithm needs more time for the filtering process of edges than Prewitt.

Prewitt and Kirsch edge detectors work well when an image has a low noise level and sharp intensity transitions. The Canny algorithm is improved in respect of the noisy edges. The smoothing is applied to the image using a Gaussian filter to reduce the noise effect. Then the intensity gradients are calculated to find the edge strength and direction like for other gradient-based edge detection algorithms. All pixels that are not the edge pixels are suppressed using the thinning process, which is applied to the gradient magnitude and called non-maxima suppression. The remaining pixels are classified using two thresholds-high and low. Edge pixels above the high threshold are assigned as strong edge, edge pixels not reaching the low threshold are discarded, and pixels with values between the two thresholds are marked as weak edges. Edge tracking and connection are realized by hysteresis and connectivity analysis, respectively.

The Laplacian filter approximates the second-order derivative. This filter has been used for edge detection as it highlights the rapid intensity change in the image and herewith the noise in those regions. The 2-D Gaussian smooth kernel of appropriate standard deviation was applied before the Laplacian filter to overcome this problem. The second derivatives are more sensitive to noise than the first ones and return edge points finding the zero crossings of the intensity image. Then the threshold is applied to minimize the false edges due to noise.

The problem of the Gaussian smoothing is that the filtering removes noise, but after blurring the edge, information can also be lost. The missing information, localization errors, and false edges are the problems of the Gaussian-based edge detectors.

Morphological edge detection combines the advantage of a Canny algorithm detecting the strength of edges and the processing the edges by morphological operations. The final result of the algorithm 
was improved by the morphological thinning. The noise and false edges can be suppressed more effectively using morphological edge detection than with the Canny algorithm.

SubPixel algorithm achieves the accurate extraction of contrast, position, orientation, and curvature of the edges. It based on the partial area effect hypothesis. The algorithm detects SubPixel features for each pixel belonging to the edges of the image. The sensitivity threshold specifies the minimum difference of intensity at both sides of a pixel to be considered as an edge.

The threshold of edge detection algorithms defines their sensitivity to the image content. When the image is noisy, has textures with high spatial frequencies of intensity changes, selection of threshold becomes a trade-off between the false and weak edges. False edges are useless and originate from noise and surface texture. Weak and strong edges are usually useful edges, but they have different characteristics. The strong edges are sharp and continuous in opposite to the weak edges. Weak and false edges can be detected as the broken segments, but weak edges are distributed more regular than false ones [42]. Canny and LoG algorithms, besides the threshold, use standard deviation that determines the filter size for smoothing. The size of the filter and smoothing degree increase with the standard deviation value. The experiments for testing the sensitivity of edge detectors were performed using threshold and standard deviation.

\subsection{A Framework of the Methodology Implementation}

The original methodology for the adaptive selection of edge detection algorithms includes six essential steps:

- Creation of the generalized set of visual characteristics (features) for the images as the criteria for the strategy;

- Selection of the set of edge detection algorithms;

- Etimation of the influence of the selected visual features of satellite images to the result of the appropriate edge detection algorithm from the defined set of algorithms as the alternatives of the strategy;

- Evaluation of the satellite image content using the set of visual characteristics of the image;

- Applying the neutrosophic WASPAS method for optimal decision making;

- Ranking of edge detection algorithms according to their suitability for the appropriate satellite image type.

For the realization of our strategy, the classical differential, morphology-based, and SubPixel edge detection methods were selected. The edge detection algorithms have to be selected for each satellite image according to the order of relevance of five criteria: contrast, roughness, density, number of different regions, size of the individual image regions. The sixth criterion, processing time, is related to the image size. Afterward, the ranking of algorithms obtained using the created methodology has to be verified with the ranking obtained in an alternative way-using the edge detection algorithms for appropriate images. The general framework of the methodology is presented in Figure 7.

The proposed methodology is universal and applicable to the selection of the edge detection algorithms for satellite images due to the adaptation of the algorithm characteristics to visual image features. 


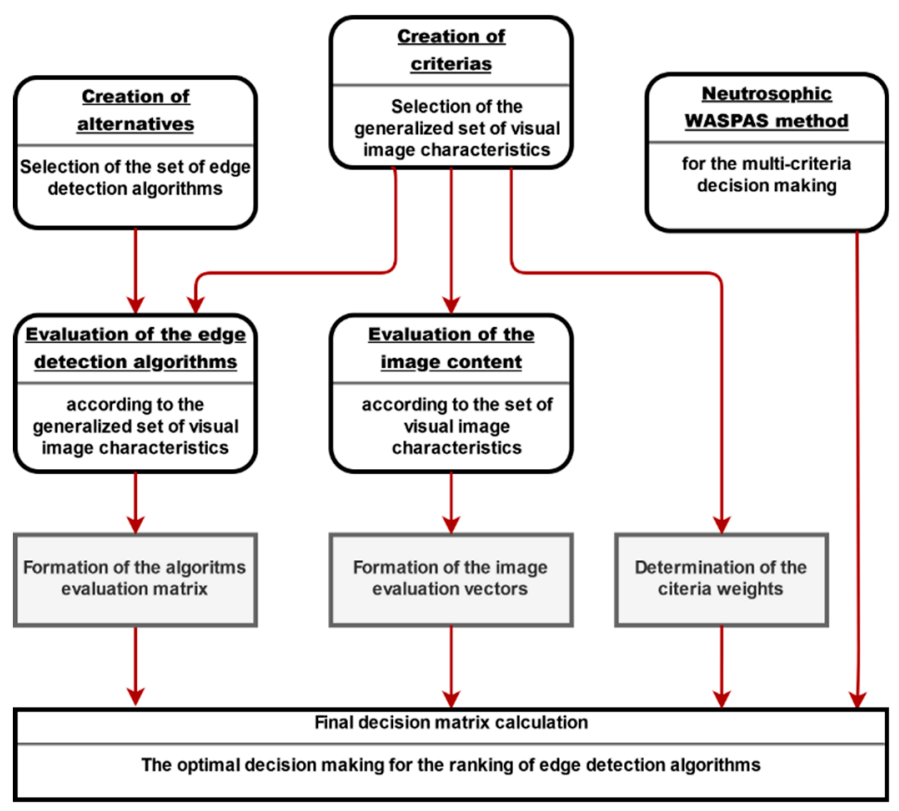

Figure 7. The general framework for the selection of the edge detection algorithms for satellite images.

\section{Selection of the MCDM Method for Data Processing and Evaluation}

In this work have been explored the problem for choosing appropriate edge detection algorithms depending on the visual image features. Generally, the WASPAS approach is quite popular for the solution of different MCDM problems. The review of the WASPAS methods application was presented in [43]. The popularity of WASPAS can be defined due to simplicity and stability. The direct subjective criteria weights evaluation method was applied for the determination of the criteria weights.

\subsection{Evaluation Method for the Criteria Weights}

Most of the currently known and applied methods of determining weights for MCDM are subjective and based on expert judgment. The subjective method described by Kendall [44]. Frequently, the subjective method is implemented for calculating criteria values $c_{i k}$ and criteria weights $w_{i}$ of importance.

In this study, we applied the direct method of criterion importance weights determination. This method has higher accuracy compared to the other ranking methods. Using the direct method for determination of the weights for criteria, the sum of the weights of each expert evaluations must be equal to $100 \%$ (or one). The criteria assessment values may be repeated [45-47].

The values $c_{i k}$ for mathematical calculations were collected by interviewing the experts. The average criterion value $\overline{c_{i}}$ was calculated by the formula:

$$
\overline{c_{i}}=\frac{\sum_{k=1}^{r} c_{i k}}{r}
$$

where $c_{i k}$ is the estimation value of the $i$ criterion by the $k$ experts, and $r$ is the number of the experts.

The weights of the criteria were calculated by dividing the sum of the criteria average values by the average value of each criterion:

$$
w_{i}=\frac{\sum_{k=1}^{r} c_{i k}}{\sum_{i=1}^{m} \sum_{k=1}^{r} c_{i k}}
$$

where $r$ is the number of experts, $m$ is the number of criteria, $c_{i k}$ is $i$ criteria by $k$ experts' determination of criteria weights. 
The total sum of weights must be equal to one:

$$
\sum_{i=1}^{m} \frac{\sum_{i=1}^{m} \bar{c}_{i}}{\bar{c}_{i}}=1
$$

Generalized experts' assessments of the importance of visual image features are presented in Table 1.

Table 1. Evaluation of the criteria importance using the direct subjective method.

\begin{tabular}{|c|c|c|c|c|c|c|}
\hline Experts & \multicolumn{6}{|c|}{ Criteria (Features) of Images } \\
\hline Expert 2 & 20 & 20 & 15 & 15 & 15 & 15 \\
\hline Expert 3 & 15 & 20 & 15 & 15 & 15 & 20 \\
\hline Expert 4 & 20 & 20 & 15 & 20 & 15 & 15 \\
\hline $\begin{array}{c}\text { Average criteria } \\
\text { values }\end{array}$ & 18 & 19 & 17 & 17 & 15 & 16 \\
\hline Criteria weights & 0.176 & 0.186 & 0.167 & 0.167 & 0.147 & 0.157 \\
\hline
\end{tabular}

In the last row of Table 1 are presented the values of criteria weights.

\subsection{MCDM WASPAS Method}

The WASPAS-SVNS extension of the WASPAS method is used to solve the mentioned MCDM problem. Previously, the WASPAS-SVNS method was applied for the problem of the real world $[17,20,21,48]$. The basis of this method is governed by the single-valued neutrosophic set. The description of the separate steps can outline the implementation of the solution to the selection of the edge detection algorithm for satellite images:

1. The first part of the initial information consists of estimates of edge detection algorithms according to selected criteria (image features). The criteria weighting coefficients are also used for the data. It can be illustrated by the initial data $x_{A i j}, i=1,2, \ldots . . m ; j=1,2 \ldots . . n$, which, in fact, is the aggregated expert assessment of the $i^{\text {th }}$ algorithm by the $j^{\text {th }}$ criterion. Accordingly, the algorithm evaluation matrix can be expressed as:

$$
X_{A}=\left[\begin{array}{cccc}
x_{A 11} & x_{A 12} & \cdots & x_{A 1 n} \\
x_{A 21} & x_{A 22} & \cdots & x_{A 2 n} \\
\vdots & \vdots & \ddots & \vdots \\
x_{A m 1} & x_{A m 2} & \cdots & x_{A m n}
\end{array}\right]
$$

2. The second part of the initial information provides the image assessment with respect to the same criteria. These assessments form the vector $X_{i}$, which has the same numbers of elements as the separate column of matrix $X_{A}$. All evaluations presented in these matrices have a linguistic form.

3. At the neutrosophication step, the initial linguistic matrix $X_{A}$ and vector $X_{i}$ are converted into the single-valued neutrosophic numbers. Consequently, the neutrosophic decision matrix $\widetilde{X_{A}^{n}}$ and vector $\widetilde{X_{i}^{n}}$ are calculated. The relationships between linguistic and neutrosophic values are applied the same as in [21].

The final decision matrix is calculated by applying the neutrosophic addition operation by summing each column of the matrix $\widetilde{X_{A}^{n}}$ with a vector $\widetilde{X_{i}^{n}}$

$$
\widetilde{X^{n}}=\widetilde{X_{A}^{n}} \bigoplus \widetilde{X_{i}^{n}}
$$


It should be mentioned, that single-valued neutrosophic number can be described by $\widetilde{N}_{1}=\left(t_{1}, i_{1}, f_{1}\right), t$ corresponds to membership degree, $i$ - to indeterminacy degree, and $t$ - to a non-membership degree. The summation of two single-valued neutrosophic numbers is governed applying the equation as follows

$$
\widetilde{N}_{1} \bigoplus \widetilde{N}_{2}=\left(t_{1}+t_{2}-t_{1} t_{2}, i_{1} i_{2}, f_{1} f_{2}\right) .
$$

4. The first decision component of the WASPAS-SVNS method is governed by the sum of the total relative importance of the alternative $i$, which is determined by the expression:

$$
\widetilde{Q}_{i}^{(1)}=\sum_{J=1}^{L \max } \widetilde{x}_{+i j}^{n} \cdot w_{+j}^{n} \bigoplus\left(\sum_{J=1}^{L \min } \widetilde{x}_{-i j}^{n} \cdot w_{-j}\right)^{c},
$$

where $\widetilde{x}_{+i j}^{n}$ and $w_{+j}$ values are associated with the criteria to be maximized and $\widetilde{x}_{-i j}^{n}$ and $w_{-j}$ values are associated with the criteria to be minimized. The multiplication of the neutrosophic variable and real crisp weight is calculated by the following expression

$$
\lambda \widetilde{N}_{1}=\left(1-\left(1-t_{1}\right)^{\lambda}, i_{1}^{\lambda}, f_{1}^{\lambda}\right), \lambda>0 .
$$

The second term of the summation (Equation (3)) consists of the complementary neutrosophic numbers, which are determined by the equation

$$
\widetilde{N}_{1}^{c}=\left(f_{1}, 1-i_{1}, t_{1}\right) .
$$

5. The second term of the optimal alternative in the WASPAS-SVNS methodology is determined by applying the formulation of the product total relative importance of the alternative $i$ and is calculated by the described expression:

$$
\widetilde{Q}_{i}^{(2)}=\prod_{j=1}^{L_{\max }}\left(\widetilde{x}_{+i j}^{n}\right)^{w_{+j}^{n}} \cdot\left(\prod_{j=1}^{L_{\min }}\left(\widetilde{x}_{-i j}^{n}\right)^{w_{-j}^{n}}\right)^{c} .
$$

The meaning of the terms of this equation is the same as in Equation (4). The multiplication of the neutrosophic numbers is governed applying the equation as follows

$$
\widetilde{N}_{1} \bigotimes \widetilde{N}_{2}=\left(t_{1} t_{2}, i_{1}+i_{2}-i_{1} i_{2}, f_{1}+f_{2}-f_{1} f_{2}\right) .
$$

6. For the final ranking of the alternatives by the applied WASPAS-SVNS method, we need to calculate final generalized criterion as follows:

$$
\widetilde{Q}_{i}=0.5 \widetilde{Q}_{i}^{(1)} \bigoplus 0.5 \widetilde{Q}_{i}^{(2)} .
$$

7. In the final step, the score function $S\left(\widetilde{Q}_{i}\right)$ is calculated for all ranking alternatives $i=1,2, \ldots, m$ by the following equation

$$
S\left(\widetilde{N}_{A}\right)=\frac{3+t_{A}-2 i_{A}-f_{A}}{4},
$$

and the final rankings of the alternatives may be formed by applying the descending order of the $S\left(\widetilde{Q}_{i}\right) i=1,2, \ldots, m$. 


\section{Results and Discussion}

This section describes a study for selecting edge detection algorithms based on the content of satellite images. The set of criteria related to the visual characteristics of the images was selected for the content evaluation of the satellite images. The influence of the selected visual characteristics of satellite images to the result of the edge detection algorithms was estimated. The edge detection algorithms were ranked according to their suitability for corresponding images using the neutrosophic WASPAS method. Ranking of algorithms obtained using the created methodology was verified with the ranking of experts using the results of the edge detection algorithms for particular images.

\subsection{Subjective Evaluation of Algorithms and Satellite Image Content}

Six edge detection algorithms were selected for the initial experiment: Prewitt, Kirsch, LoG, morphological, SubPixel. Every algorithm returned a unique edge map for the image. The content of the selected image also influences the result. Five experts were presented the edge detection results to evaluate the sensitivity (constant values, Table 2) of the edge detectors to the image content. A consistent pattern was discovered to match the edge detection algorithm to the appropriate image type. The image type is determined by its content features like roughness, density, contrast, size of individual regions, number of different regions. The time factor was estimated as an important criterion for each edge detection algorithm. Experts from Vilnius Gediminas technical university, Department of Graphical systems with at least 15 years of experience in image processing and analysis, participated in the evaluations of algorithms and images considered in the present section. These experts are also presented their opinion concerning the image visual criteria weights considered in Section 3.1. To rank the different edge detection algorithms, the 10-point Likert scale [49] was used. The experts were asked to fill the questionnaire (see Appendix A), and the mean values of the assessments were calculated. The results of the poll are summarized in Table 2 below.

Table 2. Experts' opinions in the evaluating of the algorithms.

\begin{tabular}{cccccccc}
\hline \multirow{2}{*}{ Criteria (Features) of Images } & \multirow{2}{*}{ Optimum } & \multicolumn{5}{c}{ Edge Detection Algorithms (Alternatives) } \\
\cline { 3 - 7 } & & Prewitt, $A_{\mathbf{1}}$ & Kirsch, $A_{\mathbf{2}}$ & Canny, $A_{\mathbf{3}}$ & LoG, $A_{\mathbf{4}}$ & Morphological, $A_{\mathbf{5}}$ & SubPixel, $A_{\mathbf{6}}$ \\
\hline Roughness, $C_{1}$ & $\min$ & 6 & 5 & 9 & 4 & 2 \\
\hline Density, $C_{2}$ & $\max$ & 5 & 4 & 6 & 3 & 9 \\
\hline Contrast, $C_{3}$ & $\max$ & 5 & 6 & 9 & 4 & 7 & 7 \\
\hline Processing time, $C_{4}$ & $\min$ & 9 & 4 & 5 & 8 & 6 \\
\hline Size of individual regions, $C_{5}$ & $\max$ & 3 & 5 & 8 & 4 & 9 \\
\hline Number of different regions, $C_{6}$ & $\max$ & 5 & 6 & 7 & 4 & 9 \\
\hline
\end{tabular}

Experts' opinions in the second table show that the evaluations of some algorithms are significantly different, while Canny and SubPixel were evaluated in a similar manner. However, one of the criteria may differ more, like processing time for the Canny and SubPixel algorithms. The "roughness" criterion with the optimum value min will match the smoothness feature of the image content. The value of this criterion for the morphological algorithm is the lowest compared to the values of the other algorithms. Most of the criteria of the Prewitt, Kirsch, and LoG algorithms have lower criteria values compared to the Canny, SubPixel, and morphological algorithms. However, Prewitt and LoG algorithms are fast enough. The Prewitt algorithm was included as a quick method to assess how it lags qualitatively. In terms of time, the LoG algorithm differs little from Prewitt. The Kirsch algorithm, which does not have high criteria values, is taken to test how it lags against all other selected algorithms.

All developed edge detection algorithms have their advantages and disadvantages. They generate the edge maps of different quality, and processing time also varies. The quality of edge detector results is inseparable from the visual characteristics of the image content. The criteria weights used in our methodology also differ (see Table 1). The results of the algorithms' evaluations (see Table 2) show that some algorithms have lower values for majority criteria compared to the criteria values of the other 
algorithms. However, only after conducting an experiment, we can determine which one fits better to the image content.

The second evaluation was focused on evaluating the content of the images. We have studied the most typical aspects of the satellite image content. We have selected images that have different visual features from the analyzed set of images and constructed a representative set of visual features, best characterizing satellite images.

Along with the evaluation of visual features set, it is essential to distinguish the most important satellite imagery cases, rather than investigate a large number of images. We have created a new strategy for evaluating the edge detection algorithms based on the generalized set of visual characteristics of the satellite image without actually classifying them.

Six satellite images of the different content were selected from Earth Explorer. We must assure the important characteristics of the image, such as roughness, edge density, and others must vary considerably as it will be easier to evaluate the sensitivity of the edge algorithms to the image content. Images were cropped to randomize the processing time and saved using a lossless image compression format. Satellite images used in evaluations (Figure 8): (a) "img1" is taken from dataset 201401_hillsborough_county_fl_6in_sp_cnir, saved as $1250 \times 1250$ PNG image and cropped to $600 \times 1000 \mathrm{px}$; (b) "img2" is taken from dataset 201402_georgetown_county_sc_6in_sp_cnir, saved as $1250 \times 1250$ PNG image and cropped to $616 \times 992$ px; (c) "img3" is taken from dataset 200601_hillsborough_county_fl_6in_sp_clr, saved as $1250 \times 1250$ PNG image; (d) "img4" is taken from dataset 201402_lake_county_fl_6in_sp_clr, saved as $1250 \times 1250$ PNG image and cropped to $651 \times 901$ px; (e) "img5" is taken from dataset 201402_lake_county_fl_6in_sp_clr, saved as $1250 \times 1250$ PNG image and cropped to $851 \times 1000$ px; (f) "img6" is taken from dataset 201402_lake_county_fl_6in_sp_clr, saved as $1250 \times 1250$ PNG image and cropped to $741 \times 751 \mathrm{px}$.

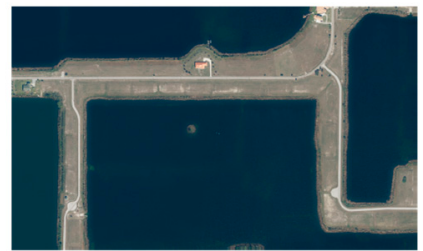

(a)

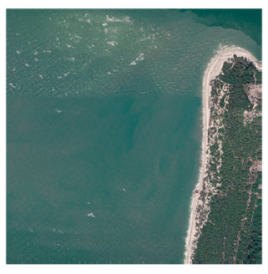

(c)

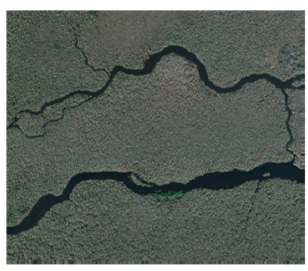

(e)

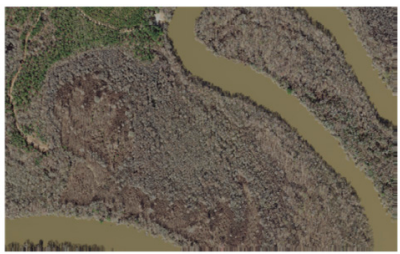

(b)

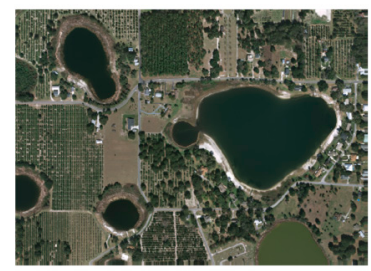

(d)

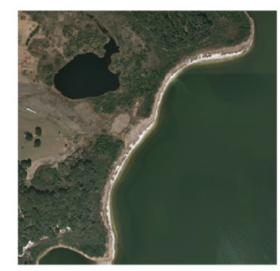

(f)

Figure 8. Satellite images used in evaluations (Landsat-7 images courtesy of the U.S. Geological Survey): (a) "img1"; (b) "img2"; (c) "img3"; (d) "img4"; (e) "img5"; (f) "img6".

The selected satellite images with appropriate visual features are presented in Figure 8a-f. 
Before starting the evaluation of the images, the respondents were explained the meaning of the criteria and a rating scale, they were provided with a questionnaire (see Appendix B) and an individual blank response table (see Table 3). The 10-point Likert scale was used for the evaluation of the image content. The respondents were asked to fill the response tables, and later the mean values of the content assessments were calculated.

Table 3. Expert opinions in the evaluation of the satellite images.

\begin{tabular}{|c|c|c|c|c|c|c|c|}
\hline \multirow{2}{*}{$\begin{array}{c}\text { Criteria (Features) of } \\
\text { Images }\end{array}$} & \multirow[b]{2}{*}{ Optimum } & \multicolumn{6}{|c|}{ Orthographic Images } \\
\hline & & $\begin{array}{l}\text { "img1", } \\
600 \times 1000\end{array}$ & $\begin{array}{l}\text { "img2", } \\
616 \times 992\end{array}$ & $\begin{array}{c}\text { "img3", } \\
1250 \times 1250\end{array}$ & $\begin{array}{l}\text { "img4", } \\
651 \times 901\end{array}$ & $\begin{array}{c}\text { "img5", } \\
851 \times 1000\end{array}$ & $\begin{array}{l}\text { "img6", } \\
741 \times 751\end{array}$ \\
\hline Roughness, $C_{1}$ & $\min$ & 9 & 1 & 4 & 3 & 1 & 4 \\
\hline Density, $C_{2}$ & $\max$ & 2 & 8 & 7 & 9 & 7 & 6 \\
\hline Contrast, $C_{3}$ & $\max$ & 8 & 4 & 6 & 7 & 6 & 7 \\
\hline Processing time, $C_{4}$ & $\min$ & 3 & 2 & 1 & 5 & 2 & 6 \\
\hline $\begin{array}{l}\text { Size of individual } \\
\text { regions, } C_{5}\end{array}$ & $\max$ & 7 & 8 & 9 & 5 & 6 & 7 \\
\hline $\begin{array}{c}\text { Number of different } \\
\text { regions, } C_{6}\end{array}$ & $\max$ & 3 & 5 & 6 & 9 & 4 & 7 \\
\hline
\end{tabular}

\subsection{Ranking of Edge Detection Algorithms}

The obtained results of the applied WASPAS-SVNS for the selection of edge detection algorithms are illustrated below. The elements of the single-valued decision matrix were calculated by neutrosophic addition operation (Equation (5)) for algorithm evaluation matrix $\widetilde{X_{A}^{n}}$ and images assessment vector $\widetilde{X_{i}^{n}}$ for image "img1" and presented in Table 4. In the same way, the decision matrices were calculated for other images ("img2", "img3", "img4", "img5", "img6").

Table 4. The decision matrix of the edge detection algorithms on the processing of the image "img1".

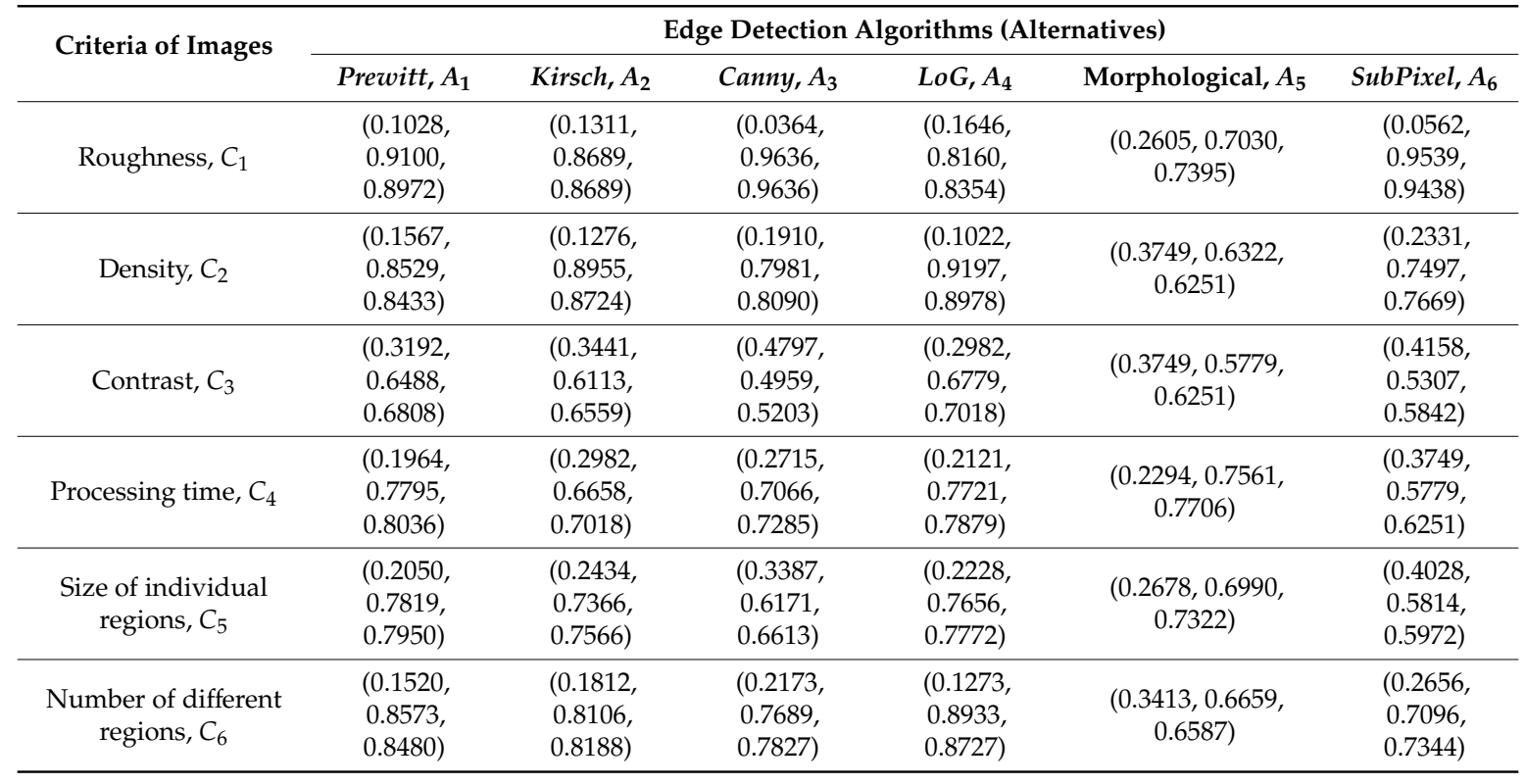

The final results of the described WASPS-SVNS methodology (steps 4-7) are presented in Table 5. We compute the first and second terms of each alternative in the WASPAS-SVNS methodology by Equations (7) and (10). Final generalized criteria $\widetilde{Q}_{i}$ were calculated by Equation (12). Likewise, the ranking of the alternatives was calculated by the application of the score function $S\left(\widetilde{N}_{A}\right)$ (Equation (13)). 
Table 5. The aggregated results provided by the WASPAS-SVNS method for "img1" processing.

\begin{tabular}{|c|c|c|c|c|c|c|}
\hline & \multicolumn{6}{|c|}{ Edge Detection Algorithms (Alternatives) } \\
\hline & Prewitt, $A_{1}$ & Kirsch, $A_{2}$ & Canny, $A_{3}$ & LoG, $A_{4}$ & Morphological, $A_{5}$ & SubPixel, $A_{6}$ \\
\hline$\widetilde{Q}_{i}^{(1)}$ & $\begin{array}{l}(0.8847 \\
0.1122 \\
0.1153)\end{array}$ & $\begin{array}{c}(0.8546 \\
0.1456 \\
0.1454)\end{array}$ & $\begin{array}{c}(0.9430 \\
0.0515 \\
0.0570)\end{array}$ & $\begin{array}{c}(0.8273 \\
0.1905 \\
0.1727)\end{array}$ & $\begin{array}{c}(0.9318,0.0673 \\
0.0682)\end{array}$ & $\begin{array}{c}(0.9332, \\
0.0583, \\
0.0668)\end{array}$ \\
\hline$\widetilde{Q}_{i}^{(2)}$ & $\begin{array}{l}(0.3263 \\
0.6734 \\
0.6737)\end{array}$ & $\begin{array}{c}(0.3260 \\
0.6763 \\
0.6740)\end{array}$ & $\begin{array}{l}(0.4111 \\
0.5716 \\
0.5889)\end{array}$ & $\begin{array}{c}(0.2888 \\
0.7280 \\
0.7112)\end{array}$ & $\begin{array}{c}(0.4469,0.5450 \\
0.5531)\end{array}$ & $\begin{array}{c}(0.4283, \\
0.5534 \\
0.5717)\end{array}$ \\
\hline$\widetilde{Q}_{i}$ & $\begin{array}{c}(0.7214 \\
0.2748 \\
0.2786)\end{array}$ & $\begin{array}{c}(0.6869 \\
0.3138 \\
0.3131)\end{array}$ & $\begin{array}{c}(0.8167 \\
0.1717 \\
0.1833)\end{array}$ & $\begin{array}{l}(0.6496 \\
0.3724 \\
0.3504)\end{array}$ & $\begin{array}{c}(0.8058,0.1916 \\
0.1942)\end{array}$ & $\begin{array}{c}(0.8046 \\
0.1797 \\
0.1954)\end{array}$ \\
\hline$S\left(\widetilde{Q}_{i}\right)$ & 0.7233 & 0.6866 & 0.8225 & 0.6386 & 0.8071 & 0.8125 \\
\hline Rank & 4 & 5 & 1 & 6 & 3 & 2 \\
\hline
\end{tabular}

It can be observed that alternative $A_{3}$ (Canny edge detection algorithm) is superior to the other mentioned alternatives. It can be concluded that for "img1" processing, the most preferred alternative is $A_{3}$. Alternatives $A_{6}$ (SubPixel algorithm) and $A_{5}$ (Morphological algorithm) are in second and third places, respectively. Alternative $A_{6}$ (LoG algorithm) is the worst variant for "img1" processing. The ranking results for all images ("img2", "img3", "img4", "img5", "img6") are presented in the next section in Table 6.

Table 6. Experts' ranking of algorithms compared to neutrosophic WASPAS method ranking.

\begin{tabular}{|c|c|c|c|c|c|c|c|c|c|}
\hline \multirow{3}{*}{$\begin{array}{l}\text { Edge Detection Algorithms } \\
\text { (Alternatives) }\end{array}$} & \multicolumn{9}{|c|}{ Satellite Images } \\
\hline & \multicolumn{2}{|c|}{ Rank by WASPAS } & \multirow[t]{2}{*}{ Experts } & \multicolumn{2}{|c|}{ Rank by WASPAS } & \multirow[t]{2}{*}{ Experts } & \multicolumn{2}{|c|}{ Rank by WASPAS } & \multirow[t]{2}{*}{ Experts } \\
\hline & & mg1" & & & mg2" & & & mg3" & \\
\hline Prewitt, $A_{1}$ & 0.7233 & 4 & 3 & 0.6944 & 5 & 5 & 0.7356 & 4 & 4 \\
\hline Kirsch, $A_{2}$ & 0.6866 & 5 & 4 & 0.6959 & 4 & 4 & 0.7284 & 5 & 5 \\
\hline Canny, $A_{3}$ & 0.8225 & 1 & 1 & 0.8001 & 3 & 3 & 0.8267 & 3 & 2 \\
\hline LoG, $A_{4}$ & 0.6386 & 6 & 5 & 0.6454 & 6 & 6 & 0.6854 & 6 & 6 \\
\hline Morphological, $A_{5}$ & 0.8071 & 3 & 6 & 0.8192 & 1 & 1 & 0.8367 & 1 & 1 \\
\hline \multirow[t]{2}{*}{ SubPixel, $A_{6}$} & 0.8125 & 2 & 2 & 0.8099 & 2 & 2 & 0.8311 & 2 & 3 \\
\hline & \multicolumn{3}{|c|}{ "img4" } & \multicolumn{3}{|c|}{ "img5" } & \multicolumn{3}{|c|}{ "img6" } \\
\hline Prewitt, $A_{1}$ & 0.8010 & 4 & 4 & 0.6513 & 5 & 5 & 0.7507 & 4 & 4 \\
\hline Kirsch, $A_{2}$ & 0.7933 & 5 & 5 & 0.6531 & 4 & 4 & 0.7321 & 5 & 5 \\
\hline Canny, $A_{3}$ & 0.8731 & 2 & 3 & 0.7713 & 3 & 3 & 0.8391 & 1 & 1 \\
\hline LoG,$A_{4}$ & 0.7614 & 6 & 6 & 0.5965 & 6 & 6 & 0.6916 & 6 & 6 \\
\hline Morphological, $A_{5}$ & 0.8723 & 3 & 2 & 0.7947 & 1 & 1 & 0.8352 & 3 & 3 \\
\hline SubPixel, $A_{6}$ & 0.8738 & 1 & 1 & 0.7841 & 2 & 2 & 0.8366 & 2 & 2 \\
\hline
\end{tabular}

\subsection{Verification of Ranked Edge Detection Methods}

The reliability of the WASPAS ranking method was verified in an alternative way. For each of the four images, six edge maps were generated varying their parameters-threshold or standard deviation and threshold-experimentally, totaling to the 24 edge maps of the relevant images. These edge maps were provided for the experts to rank them. For the experiment, the luminosity $(Y)$ component was extracted from the color satellite images from the Earth Explorer [34]. The intensity component $Y$ was processed using the selected edge detectors (first-order derivate algorithms-Prewitt, Kirsch; multistage first order derivate algorithm-Canny; second-order derivative algorithm — Laplacian of Gaussian; morphological algorithm-Canny based; SubPixel algorithm based on the hypothesis of the partial area effect).

The filtered edge maps of each image (selected region) using appropriate algorithms are presented in Figures 9-14. 


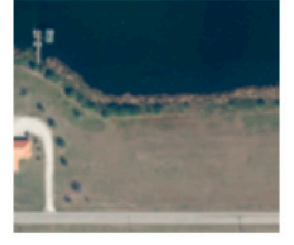

(a)

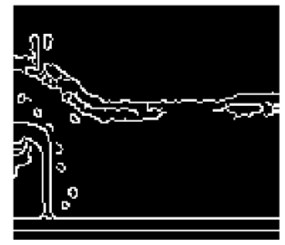

(e)

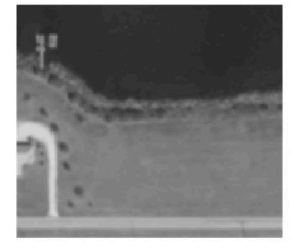

(b)

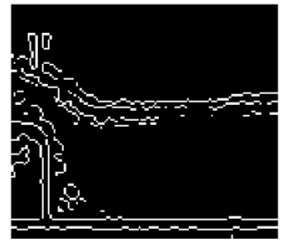

(f)

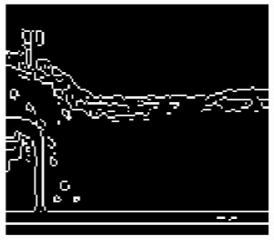

(c)

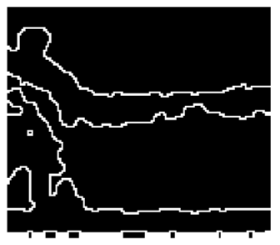

(g)

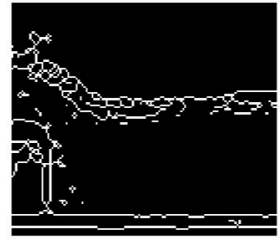

(d)

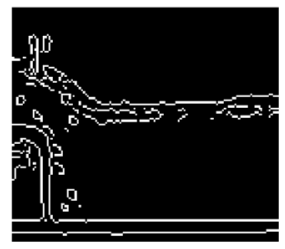

(h)

Figure 9. The region of satellite image "img1" after edge detection: (a) original color image (Landsat-7 image courtesy of the U.S. Geological Survey); (b) Y component of the original image; (c) Prewitt edge map; (d) Kirsch edge map; (e) Canny edge map; (f) LoG edge map; (g) morphological edge map; (h) SubPixel edge map.

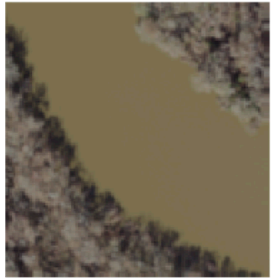

(a)

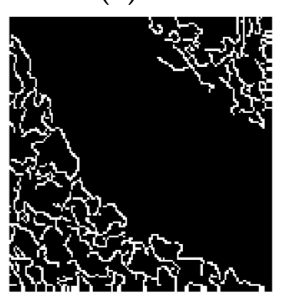

(e)

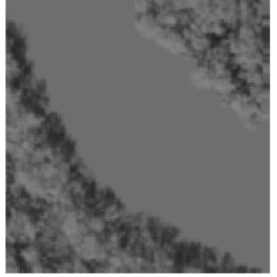

(b)

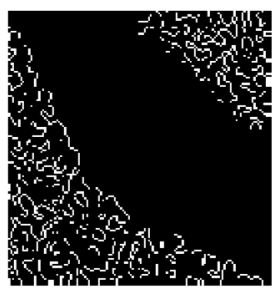

(f)

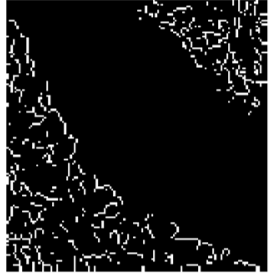

(c)

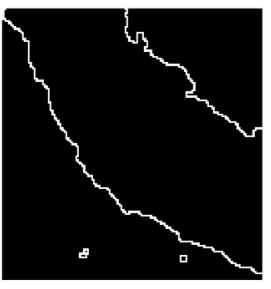

(g)

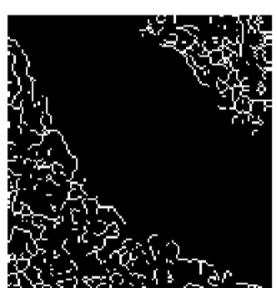

(d)

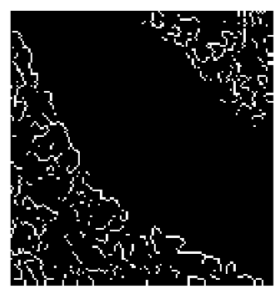

(h)

Figure 10. The region of satellite image "img2" after edge detection: (a) original color image (Landsat-7 image courtesy of the U.S. Geological Survey); (b) Y component of the original image; (c) Prewitt edge map; (d) Kirsch edge map; (e) Canny edge map; (f) LoG edge map; (g) morphological edge map; (h) SubPixel edge map. 


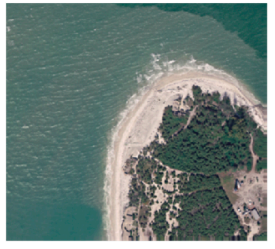

(a)

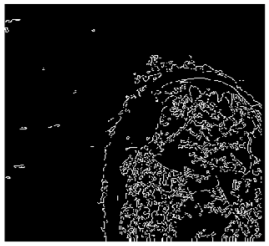

(e)

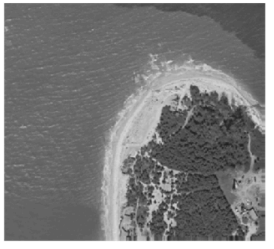

(b)

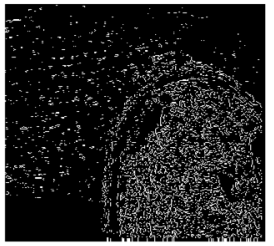

(f)

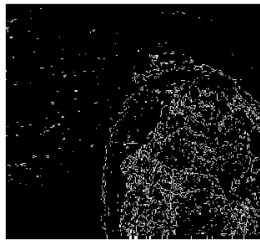

(c)

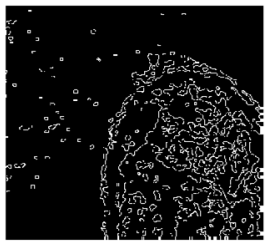

(g)

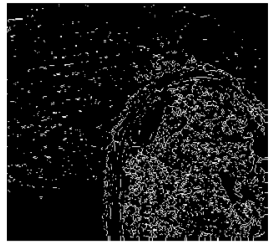

(d)

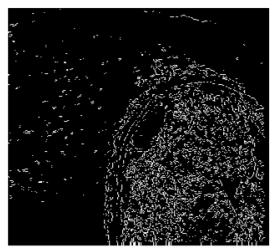

(h)

Figure 11. The region of satellite image "img3" after edge detection: (a) original color image (Landsat-7 image courtesy of the U.S. Geological Survey); (b) Y component of the original image; (c) Prewitt edge map; (d) Kirsch edge map; (e) Canny edge map; (f) LoG edge map; (g) morphological edge map; (h) SubPixel edge map.

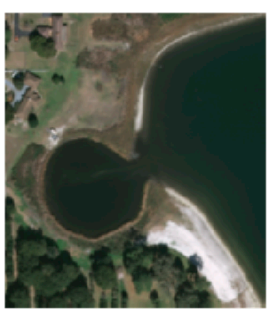

(a)

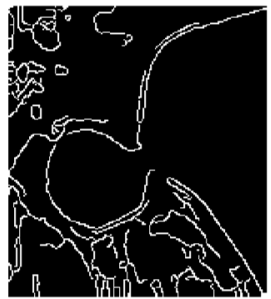

(e)

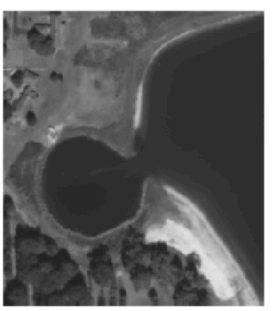

(b)

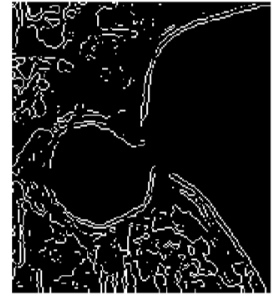

(f)

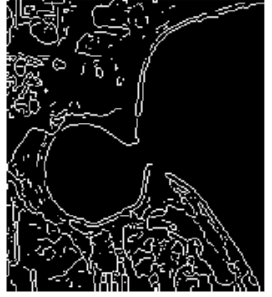

(c)

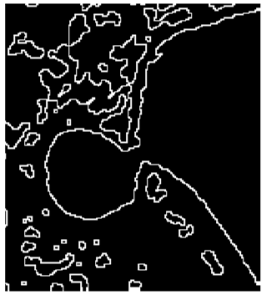

(g)

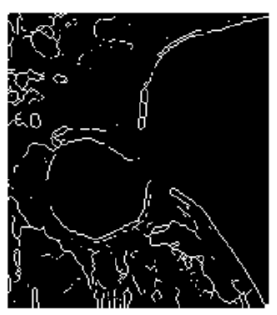

(d)

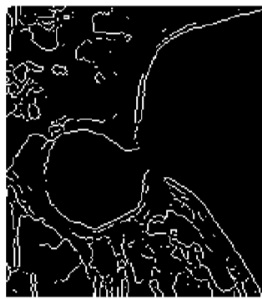

(h)

Figure 12. The region of satellite image "img4" after edge detection: (a) original color image (Landsat-7 image courtesy of the U.S. Geological Survey); (b) Y component of the original image; (c) Prewitt edge map; (d) Kirsch edge map; (e) Canny edge map; (f) LoG edge map; (g) morphological edge map; (h) SubPixel edge map. 


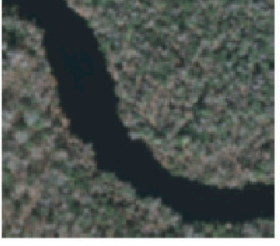

(a)

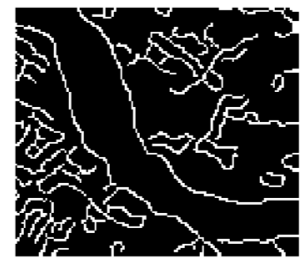

(e)

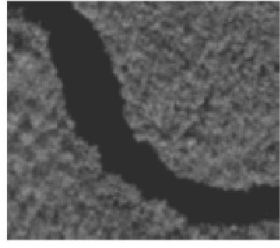

(b)

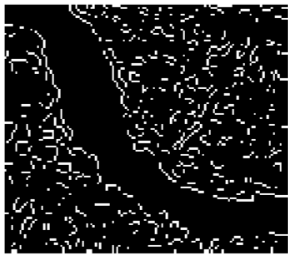

(f)

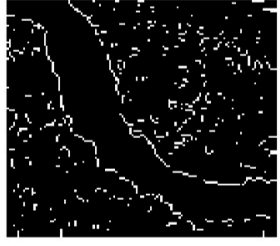

(c)

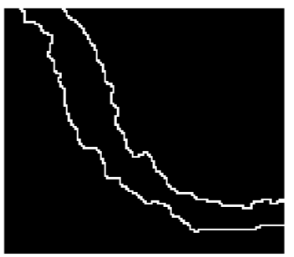

(g)

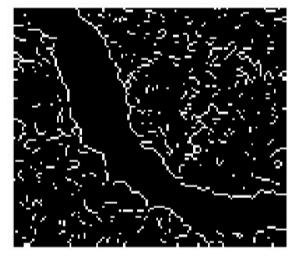

(d)

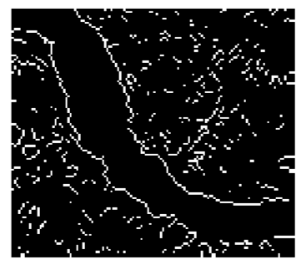

(h)

Figure 13. The region of satellite image "img5" after edge detection: (a) original color image (Landsat-7 image courtesy of the U.S. Geological Survey); (b) Y component of the original image; (c) Prewitt edge map; (d) Kirsch edge map; (e) Canny edge map; (f) LoG edge map; (g) morphological edge map; (h) SubPixel edge map.

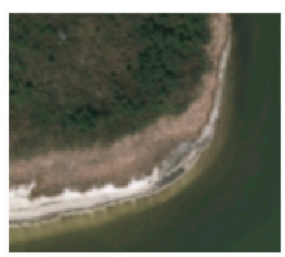

(a)

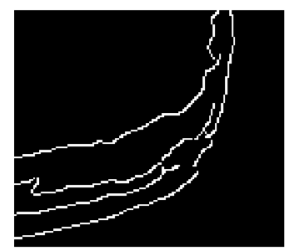

(e)

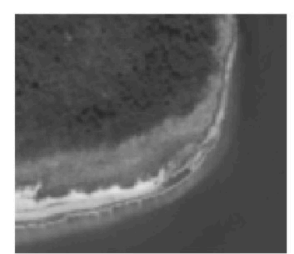

(b)

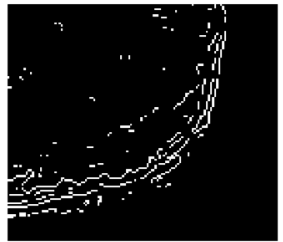

(f)

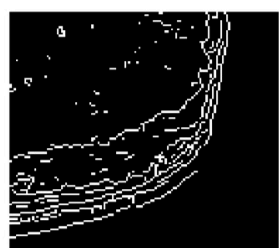

(c)

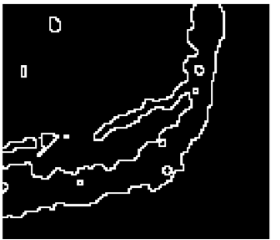

(g)

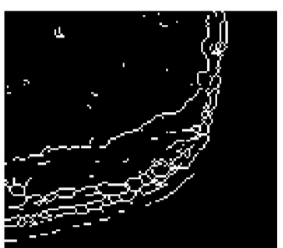

(d)

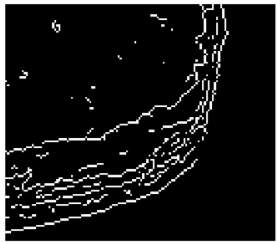

(h)

Figure 14. The region of satellite image "img6" after edge detection: (a) original color image (Landsat-7 image courtesy of the U.S. Geological Survey); (b) Y component of the original image; (c) Prewitt edge map; (d) Kirsch edge map; (e) Canny edge map; (f) LoG edge map; (g) morphological edge map; (h) SubPixel edge map.

The experts ranked the maps of each image by quality in descending order. The mean values of the ranks were calculated. After evaluation, we can see that the ranking of the algorithms does not significantly differ compared to WASPAS (Table 6).

As in WASPAS, the Canny algorithm ranks in the first place, and SubPixel is in the second place for the edge detection of the "img1". As the ranking values of the WASPAS method show, there is little difference in the result of these algorithms. The same can be observed about visual differences (Figure 9e,h). Thus, both Canny and SubPixel algorithms are suitable for images with lower roughness. The morphological algorithm was evaluated differently: it was ranked third by WASPAS and in sixth place by the experts. The ranking difference using the WASPAS method (the third place) can be 
explained by ranking higher than Prewitt, Kirsch, and LoG algorithms in many cases. The estimations of Prewitt, Kirsch, and LoG algorithms differ by only one in both cases.

The "img2" edges were best filtered by the morphological algorithm in both cases (Figure 10g). This algorithm was ranked well as it has high values of the roughness and density criteria, and the "img2" content exposes these features. The SubPixel ranks second place both by WASPAS and experts. The LoG ranks last, and it was the worst ranked by experts. Kirsch and Prewitt edge detectors are in fourth and fifth places accordingly.

For "img3" (Figure 11), the ranking is slightly different. Under the WASPAS method, the SubPixel algorithm was ranked higher than Canny due to the size of individual regions and density, and the content of "img3" has high values for these criteria. Furthermore, the weight coefficient for density is the highest.

In the case of "img4" (Figure 12), we have the same ranking using the WASPAS method and expert evaluation for all algorithms except Canny and morphological. Canny was ranked second by WASPAS and in third place by the experts. These small ranking discrepancies between Canny $(0.8731)$ and morphological algorithm (0.8723) can be explained by higher values of contrast criteria for Canny, and the "img4" content exposes this feature.

The "img5" like "img2" edges were best detected by the morphological algorithm in both cases (Figure 13g). This algorithm is suitable for images with high roughness and density criteria values. The SubPixel ranks second place both by WASPAS and experts, and Canny third place. Kirsch, Prewitt, and LoG edge detectors are in fourth, fifth, and sixth places accordingly.

In the case of "img6" (Figure 14), we have the same ranking using expert evaluation and the WASPAS method for all algorithms. The Canny algorithm was ranked first place, SubPixel is in the second, morphological in the third, and Prewitt, Kirsch, and LoG are in the fourth, fifth, and sixth places accordingly.

The additional experiment was conducted to assess the sensitivity of image content evaluation and validate the proposed methodology. Traditionally, the sensitivity analysis is applied to assess the reliability of MCDM methods. The sensitivity analysis is based on the accuracy of image evaluations, i.e., how evaluations of the respondents influence the algorithm selection.

Four new user groups, each consisting of 10 people (IT (information technologies) students, engineers, managers, and image processing experts), filled the questionnaire (see Appendix B). The "img1" was taken for sensitivity analysis. The mean values of the assessments for each considered group of the evaluators were calculated. The results of the survey are presented in Table 7 below.

Table 7. Opinions of the evaluator groups in the evaluation of the image "img1".

\begin{tabular}{cccccc}
\hline \multirow{2}{*}{ Criteria (Features) of Images } & \multirow{2}{*}{ Optimum } & \multicolumn{3}{c}{ Evaluator Groups } \\
\cline { 3 - 5 } & & Students & Engineers & Managers & Experts \\
\hline Roughness, $C_{1}$ & $\min$ & 6 & 5 & 3 & 9 \\
Density, $C_{2}$ & $\max$ & 4 & 3 & 7 & 2 \\
Contrast, $C_{3}$ & $\max$ & 7 & 9 & 1 & 8 \\
Processing time, $C_{4}$ & $\min$ & 4 & 3 & 1 & 3 \\
Size of individual regions, $C_{5}$ & $\max$ & 6 & 3 & 2 & 7 \\
Number of different regions, $C_{6}$ & $\max$ & 4 & 6 & 2 & 3 \\
\hline
\end{tabular}

The presented assessments allow make a conclusion that the opinion of the managers' group about the satellite image content varied quite perceptibly comparing to the mean values of the assessments of the fourth target group-experts (Table 7). Students' assessments are close to experts' and engineers' opinions a little differ from the experts'.

The results of the sensitivity analysis are presented in Figure 15. 


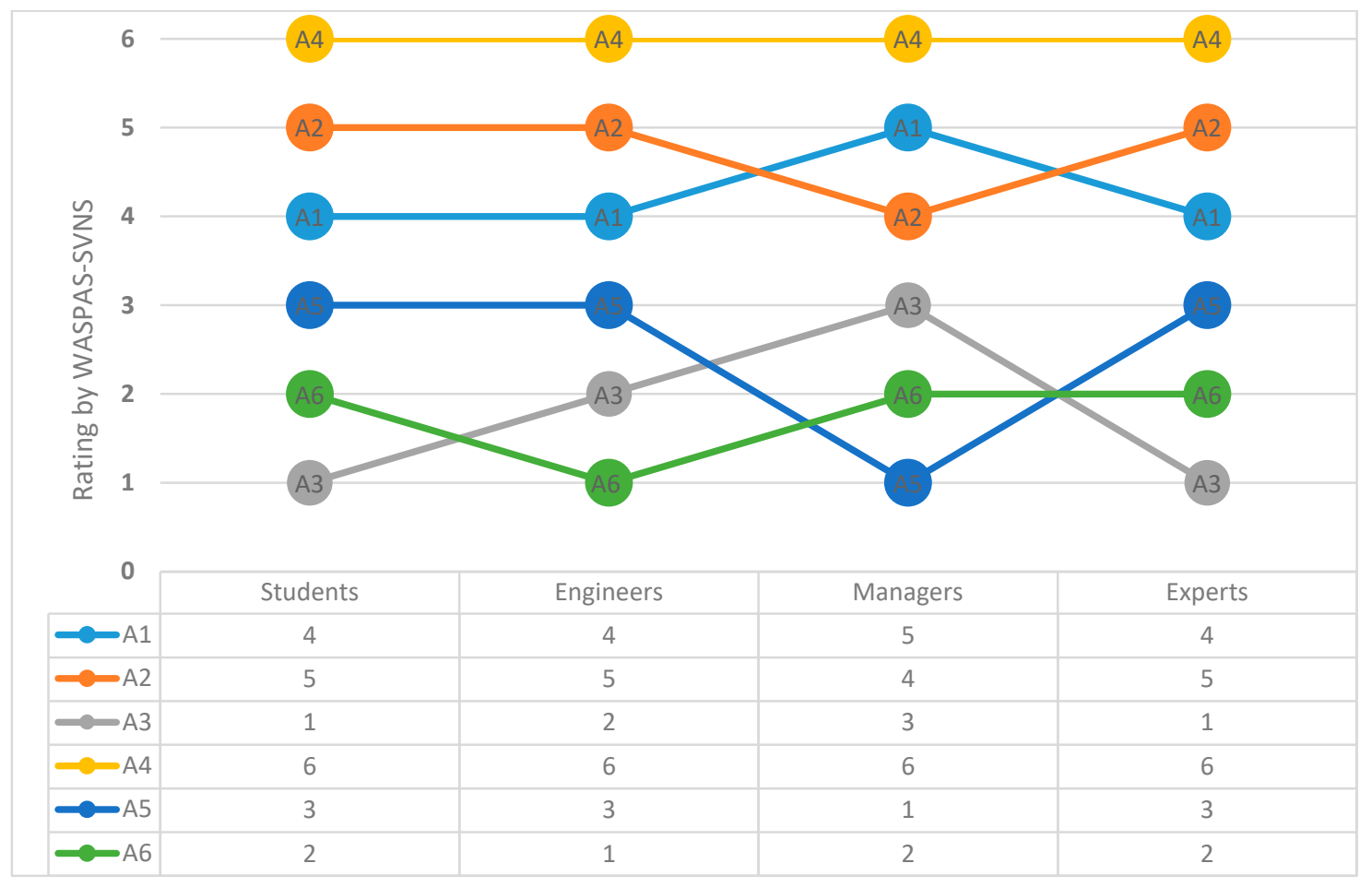

Figure 15. Rating of the six edge detection algorithms based on the different evaluation of the image content by four evaluator groups. WASPA-SVNS was used for the experiment with "img1".

The data in Figure 15 shows that the variation in the evaluators' opinions influences the results calculated using the WASPAS-SVNS method. The third alternative $\left(A_{3}\right.$, Canny's algorithm) ranks first place by students' and experts' evaluations of "img1" content. Slight fluctuations in the engineers' judgment changed the position of the A6 alternative (SubPixel's algorithm) in the ranking from second to first place. More significant deviations from the content evaluations also lead to the more significant discrepancies in the algorithm ranking; for example, $A_{5}$ (morphological algorithm) ranked first using managers' assessments of the "img1" content.

The sensitivity experiment shows the importance of proper evaluation of the image content by the experts and choosing the proper set of visual image features (criteria), influencing the algorithms' ranking. The assessment of visual content depends on the involvement of the respondent group to the field of image processing: the IT students ranked the images almost in the same order as the experts, the evaluation of engineers is slightly broader, and the evaluation of managers does not reflect the image content characteristics correctly.

\section{Conclusions and Future Work}

We proposed the novel multi-criteria decision-making methodology for the selection of the best performing edge detection algorithms for the satellite images based on their content. MCDM WASPAS-SVNS and direct criteria weights evaluation methods were used to rank the edge detection algorithms (alternatives) by visual image features. The reliability of the methodology was cross-checked with expert evaluations of edge detection results.

Because of the variety of algorithms used to detect the edges of an image, it is difficult to decide which algorithm will be appropriate for the selected image content, since several criteria determine the choice. If edge detection algorithms have high criteria values and the criteria weights are not low, their ratings for images with different content should be checked using the proposed methodology.

A set of appropriate criteria needs to be linked to the specificity and suitability of the algorithm. 
It is vital to construct a representative set of visual features that directly related to the content of the image and best characterize satellite images. This set of visual features is used as the criteria to evaluate the edge detection algorithms. The result of the edge detection algorithm depends on these criteria: roughness, density, contrast, size of individual regions, and the number of different regions. Processing time is related to image size and depends on the algorithm.

The slight differences in the ranking of the algorithms in both cases, by WASPAS and experts, are explained by the subjective evaluation of image visual features and algorithms' performance. Experts evaluated criteria weights, algorithms' sensitivity to image content, the edge map created by the appropriate algorithms, and the content of images. The correctness of the ranking is based not on the statistical evaluation of the number of samples, but on that how well the constructed set of original visual features describes the cases of the content of the characteristic satellite images. For this purpose, it must be distinguished most important satellite imagery cases. The sensitivity of evaluations of the image content is also essential. The reliability studies of MCDM methods are usually performed by sensitivity analysis but not by the statistical analysis. Sensitivity analysis allows us to detect what imperfections of user image evaluations influence the final result of the algorithms' selection. Allowing the values of each criterion of the image fluctuate within a wide range when evaluating the image by different respondents may lead to incorrect algorithm ranking. If the differences between the values of all the criteria (differences in the visual characteristics of the images) are small, the ranking of edge detection algorithms will be similar too.

Developing an expert system, we are going to include the set of criteria (visual characteristics of the images) and the evaluation ranges of each criterion along with its visual representation. This possibility would allow users to rate images of different content more accurately and effectively, as the image processing algorithms (edge detection in our case) are used not only by specialists in this field.

In this work, the classical differentiation, morphological, and subpixel edge detectors were employed, while other edge detection techniques such as multiresolution and other morphological based approaches, methods of computational intelligence for the broader set of images are to be considered for the future work. The objective criteria related to the subjective visual criteria of satellite images are to be explored.

Author Contributions: Conceptualization, R.B. and G.K.-J.; methodology, R.B., G.K.-J., F.C.; software, G.K.-J.; validation, R.B., G.K.-J., F.C.; formal analysis, R.B., G.K.-J., F.C., A.U.; investigation, G.K.-J.; resources, R.B.; data curation, G.K.-J.; writing—original draft preparation, G.K.-J.; writing—review and editing, R.B., G.K.-J., F.C., A.U.; supervision, R.B. and F.C.; project administration, R.B. and F.C. All authors have read and agreed to the published version of the manuscript.

Funding: The research received no external funding.

Conflicts of Interest: The authors declare no conflict of interest.

\section{Appendix A}

- The first questionnaire for the evaluation of the edge detection algorithms.

- Evaluate how each algorithm processes the images with low roughness.

- Evaluate how each algorithm processes the images with high density.

- Evaluate how each algorithm processes the images with high contrast.

- Evaluate how fast each algorithm processes the images.

- Evaluate how each algorithm processes the images with a large size of individual regions.

- Evaluate how each algorithm processes the images with a high number of different regions.

\section{Appendix B}

- The second questionnaire for the evaluation of the image content.

- Does the image have low roughness?

- Does the image have a high density? 
- Does the image have a high contrast?

- How fast will the image be processed according to its size?

- Does the image have a large size of individual regions?

- Does the image have a high number of different regions?

\section{References}

1. Tamura, H.; Mori, S.; Yamawaki, T. Textural Features Corresponding to Visual Perception. IEEE Trans. Syst. Man Cybern. 1978, 8, 460-473. [CrossRef]

2. Paravolidakis, V.; Ragia, L.; Moirogiorgou, K.; Zervakis, M.E. Automatic Coastline Extraction Using Edge Detection and Optimization Procedures. Geosciences 2018, 8, 407. [CrossRef]

3. Aamir, M.; Pu, Y.F.; Rahman, Z.; Tahir, M.; Naeem, H.; Dai, Q. A Framework for Automatic Building Detection from Low-Contrast Satellite Images. Symmetry 2019, 11, 3. [CrossRef]

4. Samiei, S.; Rasti, P.; Daniel, H.; Belin, E.; Richard, P.; Rousseau, D. Toward a Computer Vision Perspective on the Visual Impact of Vegetation in Symmetries of Urban Environments. Symmetry 2018, 10, 666. [CrossRef]

5. Naeem, S.; Siraj, S. A Framework to Select Edge Detection Method using Multi-criteria Decision Making. In Proceedings of the 2013 IEEE International Conference on Systems, Man, and Cybernetics, Manchester, UK, 13-16 October 2013.

6. Ziou, D.; Tabbone, S. Edge detection techniques: An overview. Pattern Recognit. Image Anal. CC Raspoznavaniye Obraz. I Anal. Izobr. 1998, 8, 537-559.

7. Li, R.; Han, D.; Dezert, J.; Yang, Y. A novel edge detector for color images based on MCDM with evidential reasoning. In Proceedings of the 2017 20th International Conference on Information Fusion (Fusion), Xi'an, China, 10-13 July 2017.

8. Papari, G.; Petkov, N. Edge and line oriented contour detection: State of the art. Image Vis. Comput. 2011, 29, 79-103. [CrossRef]

9. Ye, Q.; Gao, W.; Wang, W. A New Texture-Insensitive Edge Detection Method. In Proceedings of the 2003 Information, Communications and Signal Processing, Singapore, 15-18 December 2003. [CrossRef]

10. Zhou, X.S.; Huang, T.S. Edge-based structural features for content-based image retrieval. J. Pattern Recognit. Lett. Spec. Issue Image Video Index. Retr. Arch. 2001, 22, 457-468. [CrossRef]

11. Amadasun, M.; King, R. Texural Features Corresponding to Texural Properties. IEEE Trans. Syst. Man Cybern. 1989, 19, 1264-1274. [CrossRef]

12. Guo, X.; Asano, C.M.; Asano, A.; Kurita, T.; Li, L. Analysis of texture characteristics associated with visual complexity Perception. Opt. Rev. 2012, 19, 306-331. [CrossRef]

13. Guo, X.; Asano, C.M.; Asano, A.; Kurita, T. Visual Complexity Perception and Texture Image Characteristics. In Proceedings of the 2011 International Conference on Biometrics and Kansei Engineering, Takamatsu, Kagawa, Japan, 19-22 September 2011. [CrossRef]

14. Oliva, A.; Mack, M.L.; Shrestha, M.; Peeper, A. Identifying the Perceptual Dimensions of Visual Complexity of Scenes. In Proceedings of the 2004 26th Annual Meeting of the Cognitive Science Society, Chicago, IL, USA, 4-7 August 2004.

15. Ciocca, G.; Corchs, S.; Gasparini, F.; Bricolo, E.; Tebano, R. Does color inuence image complexity perception? Computational Color Imaging. Lect. Notes Comput. Sci. 2015, 9016, 139-148.

16. Machado, P.; Romero, J.; Nadal, M.; Santos, A.; Correia, J.; Carballal, A. Computerized measures of visual complexity. Acta Psychol. 2015, 160, 43-57. [CrossRef] [PubMed]

17. Stojčíc, M.; Zavadskas, E.K.; Pamučar, D.; Stević, Ž.; Mardani, A. Application of MCDM Methods in Sustainability Engineering: A Literature Review 2008-2018. Symmetry 2019, 11, 350. [CrossRef]

18. Khelifi, L.; Mignotte, M. A Multi-Objective Approach Based on TOPSIS to Solve the Image Segmentation Combination Problem. In Proceedings of the 2016 23rd International Conference on Pattern Recognition, Cancun, Mexico, 4-8 December 2016. [CrossRef]

19. Palevicius, V.; Burinskiene, M.; Antucheviciene, J.; Saparauskas, J. Comparative Study of Urban Area Growth: Determining the Key Criteria of Inner Urban Development. Symmetry 2019, 11, 406. [CrossRef]

20. Zavadskas, E.K.; Bausys, R.; Mazonaviciute, I. Safety evaluation methodology of urban public parks by multi-criteria decision making. Landsc. Urban Plan. 2019, 189, 372-381. [CrossRef] 
21. Bausys, R.; Cavallaro, F.; Semenas, R. Application of sustainability principles for harsh environment exploration by autonomous robot. Sustainability 2019, 11, 2518. [CrossRef]

22. Cavallaro, F.; Zavadskas, E.K.; Streimikiene, D. Concentrated solar power (CSP) hybridized systems. Ranking based on an intuitionistic fuzzy multi-criteria algorithm. J. Clean. Prod. 2018, 179, 407-416. [CrossRef]

23. Cavallaro, F.; Zavadskas, E.K.; Streimikiene, D.; Mardani, A. Assessment of concentrated solar power (CSP) technologies based on a modified intuitionistic fuzzy TOPSIS and trigonometric entropy weights. Technol. Forecast. Soc. Chang. 2019, 140, 258-270. [CrossRef]

24. Cavallaro, F.; Zavadskas, E.K.; Raslanas, S. Evaluation of combined heat and power (CHP) systems using fuzzy shannon entropy and fuzzy TOPSIS. Sustainability 2016, 8, 556. [CrossRef]

25. Xie, S.; Tu, Z. Holistically-Nested Edge Detection. Int. J. Comput. Vis. 2017, 125, 3-18. [CrossRef]

26. Stoian, A.; Poulain, V.; Inglada, J.; Poughon, V.; Derksen, D. Land Cover Maps Production with High Resolution Satellite Image Time Series and Convolutional Neural Networks: Adaptations and Limits for Operational Systems. Sustainability 2019, 11, 1986. [CrossRef]

27. Zhou, W.; Newsam, S.; Li, C.; Shao, Z. PatternNet: A benchmark dataset for performance evaluation of remote sensing image retrieval. ISPRS J. Photogramm. Remote Sens. 2018. [CrossRef]

28. Altaei, M.S.M.; Ahmed, S.M. Satellite Image Classification using Multi Features Based Descriptors. Int. Res. J. Adv. Eng. Sci. 2018, 3, 87-94.

29. Antun, V.; Renna, F.; Poon, C.; Adcock, B.; Hansen, A.C. On instabilities of deep learning in image reconstruction-Does AI come at a cost? arXiv 2019, arXiv:1902.05300v1.

30. Guitouni, A.; Martel, J.-M. Tentative guidelines to help choosing an appropriate MCDA method. Eur. J. Oper. Res. 1998, 109, 501-521. [CrossRef]

31. Zavadskas, E.K.; Turskis, Z.; Antucheviciene, J.; Zakarevicius, A. Optimization of Weighted Aggregated Sum Product Assessment. Syst. Eng. Comput. Technol. 2012, 122, 3-6. [CrossRef]

32. Ciocca, G.; Corchs, S.; Gasparini, F. Complexity Perception of Texture Images. In Proceedings of the 2015 International Conference on Image Analysis and Processing ICIAP 2015: New Trends in Image Analysis and Processing, Genoa, Italy, 7-8 September 2015; pp. 119-126.

33. Haralick, R.M. Statistical and structural approaches to texture. Proc. IEEE 1979, 67, 786-804. [CrossRef]

34. Earth Explorer. Available online: https://earthexplorer.usgs.gov/ (accessed on 19 March 2017).

35. Liu, X.M.; Wang, C.; Yao, H.; Zhang, L. The scale of edges. In Proceedings of the 2012 IEEE Conference on Computer Vision and Pattern Recognition, Providence, RI, USA, 16-21 June 2012. [CrossRef]

36. Zhang, X.; Yang, K.; Hao, B. Cell-Edge Detection Method Based on Canny Algorithm and Mathematical Morphology. In Proceedings of the 2010 3rd International Congress on Image and Signal Processing, Yantai, China, 16-18 October 2010. [CrossRef]

37. Prewitt, J.M. Object enhancement and extraction. Pict. Process. Psychopictorics 1970, 10, 15-19.

38. Kirsch, R. Computer determination of the constituent structure of biological images. Comput. Biomed. Res. Int. J. 1971, 4, 315-328. [CrossRef]

39. Canny, J. A computational approach to edge detection. IEEE Trans. Pattern Anal. Mach. Intell. 1986, 6, 679-698. [CrossRef]

40. Marr, D.; Hildreth, E. Theory of edge detection. Proc. R. Soc. Lond. Ser. B Biol. Sci. 1980, 207, $187-217$. [CrossRef]

41. Trujillo-Pino, A.; Krissian, K.; Alemán-Flores, M.; Santana-Cedrés, D. Accurate subpixel edge location based on partial area effect. Image Vis. Comput. 2013, 31, 72-90. [CrossRef]

42. Zhang, F.; Huang, X.; Cheng, X.; Li, D. Edge extraction algorithm based on linear perception enhancement. Int. Arch. Photogramm. Remote Sens. Spat. Inf. Sci. 2008, XXXVII, 497-501.

43. Mardani, A.; Nilashi, M.; Zakuan, N.; Loganathan, N.; Soheilirad, S.; Saman, M.Z.M.; Ibrahimb, O. A systematic review and meta-Analysis of SWARA and WASPAS methods: Theory and applications with recent fuzzy developments. Appl. Soft Comput. 2017, 57, 265-292. [CrossRef]

44. Kendall, M.G. Rank Correlation Methods, 4th ed.; Griffin: London, UK, 1970; pp. 60-95.

45. Zavadskas, E.K.; Podvezko, V. Integrated Determination of Objective Criteria Weights in MCDM. Int. J. Inf. Technol. Decis. Mak. 2016, 15, 267-283. [CrossRef]

46. Zavadskas, E.K.; Ustinovichius, L.; Turskis, Z.; Shevchenko, G. Application of Verbal Methods to MultiAttribute Comparative Analysis of Investments Risk Alternatives in Construction. Comput. Model. New Technol. 2008, 12, 30-37. 
47. Zavadskas, E.K.; Vilutiene, T.A. Multiple Criteria Evaluation of Multi-Family Apartment Blocks Maintenance Contractor: I-A Model for Maintenance Contractor Evaluation and Determination of Its Selection Criteria. Build. Environ. 2006, 41, 621-632. [CrossRef]

48. Zavadskas, E.K.; Bausys, R.; Lazauskas, M. Sustainable assessment of alternative sites for the construction of a waste incineration plant by applying WASPAS method with single-valued neutrosophic set. Sustainability 2015, 7, 15923-15936. [CrossRef]

49. Likert Scale. Available online: https://www.britannica.com/topic/Likert-Scale (accessed on 29 March 2017).

(C) 2020 by the authors. Licensee MDPI, Basel, Switzerland. This article is an open access article distributed under the terms and conditions of the Creative Commons Attribution (CC BY) license (http://creativecommons.org/licenses/by/4.0/). 